\title{
A Characteristic Difference Scheme for Time-Fractional Heat Equations Based on the Crank-Nicholson Difference Schemes
}

\author{
Ibrahim Karatay and Serife R. Bayramoglu \\ Department of Mathematics, Fatih University, Buyukcekmece, 34500 Istanbul, Turkey \\ Correspondence should be addressed to Ibrahim Karatay, ikaratay@fatih.edu.tr
}

Received 25 April 2012; Revised 27 August 2012; Accepted 27 August 2012

Academic Editor: Dumitru Băleanu

Copyright (C) 2012 I. Karatay and S. R. Bayramoglu. This is an open access article distributed under the Creative Commons Attribution License, which permits unrestricted use, distribution, and reproduction in any medium, provided the original work is properly cited.

We consider the numerical solution of a time-fractional heat equation, which is obtained from the standard diffusion equation by replacing the first-order time derivative with Riemann-Liouville fractional derivative of order $\alpha$, where $0<\alpha<1$. The main purpose of this work is to extend the idea on Crank-Nicholson method to the time-fractional heat equations. We prove that the proposed method is unconditionally stable, and the numerical solution converges to the exact one with the order $O\left(\tau^{2}+h^{2}\right)$. Numerical experiments are carried out to support the theoretical claims.

\section{Introduction}

Fractional calculus is one of the most popular subjects in many scientific areas for decades. Many problems in applied science, physics and engineering are modeled mathematically by the fractional partial differential equations (FPDEs). We can see these models adoption in viscoelasticity $[1,2]$, finance $[3,4]$, hydrology [5, 6], engineering $[7,8]$, and control systems [9-11]. FPDEs may be investigated into two fundamental types: time-fractional differential equations and space-fractional differential equations.

Several different methods have been used for solving FPDEs. For the analytical solutions to problems, some methods have been proposed: the variational iteration method $[12,13]$, the Adomian decomposition method [13-16], as well as the Laplace transform and Fourier transform methods $[17,18]$.

On the other hand, numerical methods which based on a finite-difference approximation to the fractional derivative, for solving FDPEs [19-24], have been proposed. A practical numerical method for solving multidimensional fractional partial differential equations, using a variation on the classical alternating-directions implicit (ADI) Euler method, 
is presented in [25]. Many finite-difference approximations for the FPDEs are only firstorder accurate. Some second-order accurate numerical approximations for the space-fractional differential equations were presented in [26-28]. Here, we propose a Crank-Nicholson-type method for time-fractional differential heat equations with the accuracy of order $O\left(\tau^{2}+h^{2}\right)$.

In this work, we consider the following time-fractional heat equation:

$$
\begin{gathered}
\frac{\partial_{M}^{\alpha} u(t, x)}{\partial t^{\alpha}}=\frac{\partial^{2} u(t, x)}{\partial x^{2}}+f(t, x), \quad(0<x<1,0<t<1), \\
u(0, x)=r(x), \quad 0 \leq x \leq 1, \\
u(t, 0)=0, \quad u(t, 1)=0, \quad 0 \leq t \leq 1 .
\end{gathered}
$$

Here, the term $\partial_{M}^{\alpha} u(t, x) / \partial t^{\alpha}$ denotes $\alpha$-order-modified Riemann-Liouville fractional derivative [29] given with the formula:

$$
\frac{\partial_{M}^{\alpha} u(t, x)}{\partial t^{\alpha}}= \begin{cases}\frac{1}{\Gamma(1-\alpha)} \frac{\partial}{\partial t} \int_{0}^{t} \frac{u(s, x)-u(0, x)}{(t-s)^{\alpha}} d s, & \text { if } 0<\alpha<1 \\ \frac{\partial}{\partial t} u(t, x), & \text { if } \alpha=1\end{cases}
$$

where $\Gamma(\cdot)$ is the Gamma function.

Remark 1.1. If $r(x)=0$, then the Riemann-Liouville and the modified Riemann-Liouville fractional derivatives are identical, since the Riemann-Liouville derivative is given by the following formula:

$$
\frac{\partial^{\alpha} u(t, x)}{\partial t^{\alpha}}= \begin{cases}\frac{1}{\Gamma(1-\alpha)} \frac{\partial}{\partial t} \int_{0}^{t} \frac{u(s, x)}{(t-s)^{\alpha}} d s, & \text { if } 0<\alpha<1 \\ \frac{\partial}{\partial t} u(t, x), & \text { if } \alpha=1\end{cases}
$$

If $r(x)$ is nonzero, then there are some problems about the existence of the solutions for the heat equation (1.1). To rectify the situation, two main approaches can be used: the modified Riemann-Liouville fractional derivative can be used [29] or the initial condition should be modified [30]. We chose the first approach in our work.

\section{Discretization of the Problem}

In this section, we introduce the basic ideas for the numerical solution of the time-fractional heat equation (1.1) by Crank-Nicholson difference scheme.

For some positive integers $M$ and $N$, the grid sizes in space and time for the finitedifference algorithm are defined by $h=1 / M$ and $\tau=1 / N$, respectively. The grid points in the space interval $[0,1]$ are the numbers $x_{i}=i h, i=0,1,2, \ldots, M$, and the grid points in the time interval $[0,1]$ are labeled $t_{n}=n \tau, n=0,1,2, \ldots, N$. The values of the functions $U$ and $f$ at the grid points are denoted $U_{i}^{n}=U\left(t_{n}, x_{i}\right)$ and $f_{i}^{n}=f\left(t_{n}, x_{i}\right)$, respectively. 
As in the classical Crank-Nicholson difference scheme, we will obtain a discrete approximation to the fractional derivative $\partial^{\alpha} U(t, x) / \partial t^{\alpha}$ at $\left(t_{n+(1 / 2)}, x_{i}\right)$. Let

$$
H(t, x)=\frac{1}{\Gamma(1-\alpha)} \int_{0}^{t} \frac{u(s, x)-u(0, x)}{(t-s)^{\alpha}} d s
$$

Then, we have

$$
\frac{\partial^{\alpha} U\left(t_{n+1 / 2}, x_{i}\right)}{\partial t^{\alpha}}=\frac{\partial}{\partial t} H\left(t_{n+1 / 2}, x_{i}\right)=\frac{H\left(t_{n+1}, x_{i}\right)-H\left(t_{n}, x_{i}\right)}{\tau}+O\left(\tau^{2}\right) .
$$

Now, we will find the approximations for $H\left(t_{n+1}, x_{i}\right)$ and $H\left(t_{n}, x_{i}\right)$ :

$$
\begin{aligned}
H\left(t_{n+1}, x_{i}\right)= & \frac{1}{\Gamma(1-\alpha)} \int_{0}^{t_{n+1}} \frac{u\left(s, x_{i}\right)-u\left(0, x_{i}\right)}{\left(t_{n+1}-s\right)^{\alpha}} d s \\
= & \frac{1}{\Gamma(1-\alpha)} \sum_{j=1}^{n+1} \int_{(j-1) \tau}^{j \tau} \frac{u\left(s, x_{i}\right)}{\left(t_{n+1}-s\right)^{\alpha}} d s-u\left(0, x_{i}\right) \frac{((n+1) \tau)^{1-\alpha}}{\Gamma(2-\alpha)} \\
= & \frac{1}{\Gamma(1-\alpha)} \sum_{j=1}^{n+1} \int_{(j-1) \tau}^{j \tau}\left[\frac{\left(s-t_{j}\right)}{-\tau} U_{i}^{j-1}+\frac{\left(s-t_{j-1}\right)}{\tau} U_{i}^{j}+O\left(\tau^{2}\right)\right] \frac{1}{\left(t_{n+1}-s\right)^{\alpha}} d s \\
& \quad-U_{i}^{0} \frac{((n+1) \tau)^{1-\alpha}}{\Gamma(2-\alpha)} \\
= & \tau \sum_{j=0}^{n}\left(a_{j}-j b_{j}\right) U_{i}^{n-j}-\tau \sum_{j=0}^{n}\left(a_{j}-(j+1) b_{j}\right) U_{i}^{n-j+1}-U_{i}^{0} \frac{((n+1) \tau)^{1-\alpha}}{\Gamma(2-\alpha)}+R_{n+1},
\end{aligned}
$$

where

$$
\begin{aligned}
R_{n+1} & =\frac{1}{\Gamma(1-\alpha)} \sum_{j=1}^{n+1} \int_{(j-1) \tau}^{j \tau} O\left(\tau^{2}\right) \frac{d s}{\left(t_{n+1}-s\right)^{\alpha}} \\
& =\frac{1}{(1-\alpha) \Gamma(1-\alpha)} O\left(\tau^{2}\right) \sum_{j=1}^{n+1}\left[(n-j+2)^{1-\alpha}-(n-j+1)^{1-\alpha}\right] \tau^{1-\alpha} \\
& =\frac{1}{\Gamma(2-\alpha)}(n+1)^{1-\alpha} O\left(\tau^{3-\alpha}\right) .
\end{aligned}
$$


Similarly, we can obtain

$$
\begin{aligned}
H\left(t_{n}, x_{i}\right) & =\frac{1}{\Gamma(1-\alpha)} \int_{0}^{t_{n}} \frac{u\left(s, x_{i}\right)-u\left(0, x_{i}\right)}{\left(t_{n}-s\right)^{\alpha}} d s \\
& =\tau \sum_{j=1}^{n}\left(a_{j-1}-(j-1) b_{j-1}\right) U_{i}^{n-j}-\tau \sum_{j=1}^{n}\left(a_{j-1}-j b_{j-1}\right) U_{i}^{n-j+1}-U_{i}^{0} \frac{(n \tau)^{1-\alpha}}{\Gamma(2-\alpha)}+R_{n},
\end{aligned}
$$

where $R_{n}=(1 / \Gamma(2-\alpha)) n^{1-\alpha} O\left(\tau^{3-\alpha}\right)$ and

$$
a_{j}=\frac{\tau^{-\alpha}}{(2-\alpha) \Gamma(1-\alpha)}\left[(j+1)^{2-\alpha}-j^{2-\alpha}\right], \quad b_{j}=\frac{\tau^{-\alpha}}{(1-\alpha) \Gamma(1-\alpha)}\left[(j+1)^{1-\alpha}-j^{1-\alpha}\right] .
$$

Then, we can write the following approximation:

$$
\begin{aligned}
\frac{\partial^{\alpha} U\left(t_{n+1 / 2}, x_{i}\right)}{\partial t^{\alpha}} & =\frac{H\left(t_{n+1}, x_{i}\right)-H\left(t_{n}, x_{i}\right)}{\tau}+O\left(\tau^{2}\right) \\
& =q_{n} U_{i}^{0}+\sum_{j=0}^{n} p_{j} U_{i}^{n+1-j}+\frac{R^{n+1}-R^{n}}{\tau}+O\left(\tau^{2}\right) \\
& =q_{n} U_{i}^{0}+\sum_{j=0}^{n} p_{j} U_{i}^{n+1-j}+\frac{1}{\Gamma(2-\alpha)}\left[(n+1)^{1-\alpha}-n^{1-\alpha}\right] O\left(\tau^{2-\alpha}\right)+O\left(\tau^{2}\right) \\
& =q_{n} U_{i}^{0}+\sum_{j=0}^{n} p_{j} U_{i}^{n+1-j}+\frac{1}{\Gamma(2-\alpha)}\left[\frac{(n+1)^{1-\alpha}-n^{1-\alpha}}{\tau}\right] O\left(\tau^{3-\alpha}\right)+O\left(\tau^{2}\right) \\
& =q_{n} U_{i}^{0}+\sum_{j=0}^{n} p_{j} U_{i}^{n+1-j}+\frac{1}{\Gamma(2-\alpha)}\left[\frac{(\tau(n+1))^{1-\alpha}-(\tau n)^{1-\alpha}}{\tau}\right] O\left(\tau^{2}\right)+O\left(\tau^{2}\right),
\end{aligned}
$$

where

$$
\begin{aligned}
& q_{0}=3 a_{0}-a_{1}+2 b_{1}-2 b_{0}, \\
& q_{n}=a_{n}-a_{n-1}+(n-1) b_{n-1}-(n+1) b_{n}, \quad \text { for } 1 \leq n \leq N-1, \\
& p_{0}=b_{0}-a_{0} \\
& p_{1}=2 a_{0}-a_{1}+2 b_{1}-b_{0}, \\
& p_{j}=\left(-a_{j-2}+2 a_{j-1}-a_{j}\right)+(j-2) b_{j-2}-(2 j-1) b_{j-1}+(j+1) b_{j}, \quad \text { for } j \geq 2 .
\end{aligned}
$$

On the other hand, using the mean-value theorem, we get

$$
\frac{(\tau(n+1))^{1-\alpha}-(\tau n)^{1-\alpha}}{\tau}=f^{\prime}(c)=\text { constant }
$$


where $f(x)=x^{1-\alpha}$ and $t_{n}<c<t_{n+1}$. So, we obtain the following second-order approximation for the modified Riemann-Liouville derivative:

$$
\begin{aligned}
\frac{\partial^{\alpha} U\left(t_{n+(1 / 2)}, x_{i}\right)}{\partial t^{\alpha}} & =\frac{H\left(t_{n+1}, x_{i}\right)-H\left(t_{n}, x_{i}\right)}{\tau}+O\left(\tau^{2}\right) \\
& =q_{n} U_{i}^{0}+\sum_{j=0}^{n} p_{j} U_{i}^{n+1-j}+O\left(\tau^{2}\right) .
\end{aligned}
$$

\section{Crank-Nicholson Difference Scheme}

Using the approximation above, we obtain the following difference scheme which is accurate of order $O\left(\tau^{2}+h^{2}\right)$ :

$$
\begin{gathered}
q_{n} U_{i}^{0}+\sum_{j=0}^{n} p_{j} U_{i}^{n+1-j}-\left[\frac{U_{i+1}^{n+1}-2 U_{i}^{n+1}+U_{i-1}^{n+1}}{2 h^{2}}+\frac{U_{i+1}^{n}-2 U_{i}^{n}+U_{i-1}^{n}}{2 h^{2}}\right] \\
=f\left(t_{n}+\frac{\tau}{2}, x_{i}\right), \quad 0 \leq n \leq N-1, \quad 1 \leq i \leq M-1, \\
U_{i}^{0}=r\left(x_{i}\right), \quad 1 \leq i \leq M-1, \\
U_{0}^{n}=0, \quad U_{M}^{n}=0, \quad 0 \leq n \leq N .
\end{gathered}
$$

We can arrange the system above to obtain

$$
\begin{gathered}
\left(-\frac{1}{2 h^{2}}\right)\left(U_{i+1}^{n+1}+U_{i+1}^{n}\right)+q_{n} U_{i}^{0}+\sum_{j=0}^{n} p_{j} U_{i}^{n+1-j}+\left(-\frac{1}{2 h^{2}}\right)\left(U_{i-1}^{n+1}+U_{i-1}^{n}\right) \\
=f\left(t_{n}+\frac{\tau}{2}, x_{i}\right), \quad 0 \leq n \leq N-1,1 \leq i \leq M-1, \\
U_{i}^{0}=r\left(x_{i}\right), \quad 1 \leq i \leq M-1, \\
U_{0}^{n}=0, \quad U_{M}^{n}=0, \quad 0 \leq n \leq N .
\end{gathered}
$$

The difference scheme above can be written in matrix form:

$$
A U_{i+1}+B U_{i}+A U_{i-1}=\varphi_{i}
$$

where $\varphi_{i}=\left[\varphi_{i}^{0}, \varphi_{i}^{1}, \varphi_{i}^{2}, \ldots, \varphi_{i}^{N}\right]^{T}, \varphi_{i}^{0}=r\left(x_{i}\right), \varphi_{i}^{n}=f\left(t_{n+1 / 2}, x_{i}\right), 1 \leq n \leq N, 1 \leq i \leq M$, and $U_{i}=\left[U_{i}^{0}, U_{i}^{1}, U_{i}^{2}, \ldots, U_{i}^{N}\right]^{T}$. 
Here, $A_{(N+1) \times(N+1)}$ and $B_{(N+1) \times(N+1)}$ are the matrices of the form

$$
\begin{aligned}
& A=\left(-\frac{1}{2 h^{2}}\right)\left[\begin{array}{ccccc}
0 & & & & \\
1 & 1 & & & \\
& 1 & 1 & & \\
& & \ddots & \ddots & \\
& & & 1 & 1
\end{array}\right]
\end{aligned}
$$

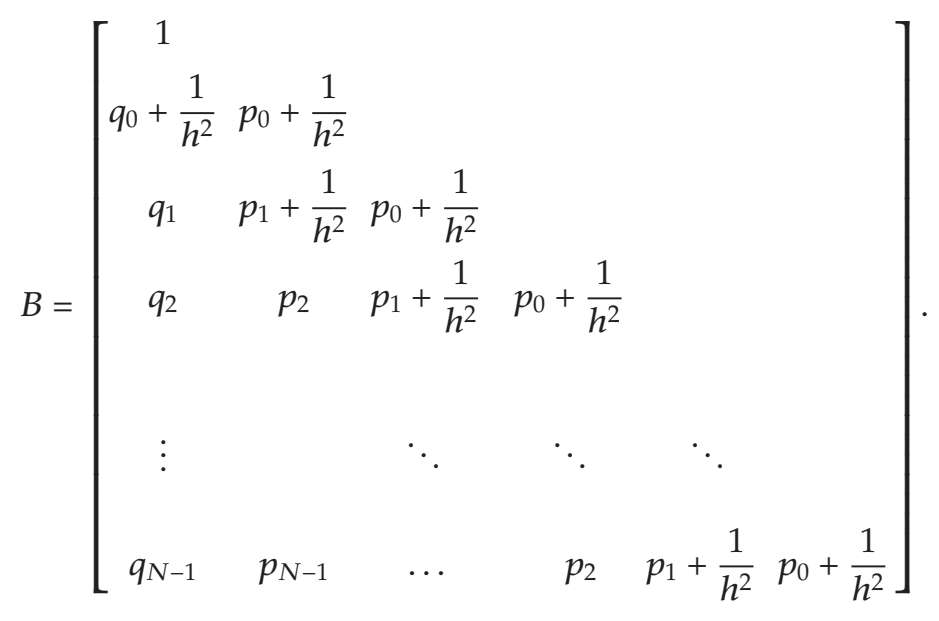

We note that the unspecified entries are zero at the matrices above.

Using the idea on the modified Gauss-Elimination method, we can convert (3.3) into the following form:

$$
U_{i}=\alpha_{i+1} U_{i+1}+\beta_{i+1}, \quad i=M-1, \ldots, 2,1,0
$$

This way, the two-step form of difference schemes in (3.3) is transformed to one-step method as in (3.5).

Now, we need to determine the matrices $\alpha_{i+1}$ and $\beta_{i+1}$ satisfying the last equality. Since $U_{0}=\alpha_{1} U_{1}+\beta_{1}=0$, we can select $\alpha_{1}=O_{(N+1) \times(N+1)}$ and $\beta_{1}=O_{(N+1) \times 1}$. Combining the equalities $U_{i}=\alpha_{i+1} U_{i+1}+\beta_{i+1}$ and $U_{i-1}=\alpha_{i} U_{i}+\beta_{i}$ and the matrix equation (3.3), we have

$$
\left(A+B \alpha_{i+1}+A \alpha_{i} \alpha_{i+1}\right) U_{i+1}+\left(B \beta_{i+1}+A \alpha_{i} \beta_{i+1}+A \beta_{i}\right)=\varphi_{i}
$$

Then, we write

$$
\begin{gathered}
A+B \alpha_{i+1}+A \alpha_{i} \alpha_{i+1}=0 \\
B \beta_{i+1}+A \alpha_{i} \beta_{i+1}+A \beta_{i}=\varphi_{i}
\end{gathered}
$$

where $1 \leq i \leq M-1$. 
So, we obtain the following pair of formulas:

$$
\begin{gathered}
\alpha_{i+1}=-\left(B+A \alpha_{i}\right)^{-1} A, \\
\beta_{i+1}=\left(B+A \alpha_{i}\right)^{-1}\left(\varphi_{i}-A \beta_{i}\right),
\end{gathered}
$$

where $1 \leq i \leq M-1$.

\section{Stability of the Method}

The stability analysis is done by using the analysis of the eigenvalues of the iteration matrix $\alpha_{i}(1 \leq i \leq M)$ of the scheme (3.5).

Let $\rho(A)$ denote the spectral radius of a matrix $A$, that is, the maximum of the absolute value of the eigenvalues of the matrix $A$.

We will prove that $\rho\left(\alpha_{i}\right)<1,(1 \leq i \leq M)$, by induction.

Since $\alpha_{1}$ is a zero matrix $\rho\left(\alpha_{1}\right)=0<1$.

Moreover, $\alpha_{2}=-B^{-1} A, \rho\left(\alpha_{2}\right)=\rho\left(-B^{-1} A\right)=\frac{-1}{1 / h^{2}+p_{0}} \cdot \frac{-1}{2 h^{2}}=\frac{1 / h^{2}}{2\left(1 / h^{2}+p_{0}\right)}$, since $\alpha_{2}$ is of the form

$$
\begin{aligned}
& \alpha_{2}=\left[\begin{array}{ccccc}
0 & & & & \\
* & \frac{1 / h^{2}}{2\left(1 / h^{2}+p_{0}\right)} & & & \\
* & * & \frac{1 / h^{2}}{2\left(1 / h^{2}+p_{0}\right)} & \\
& & & \ddots & \\
* & * & * & & \frac{1 / h^{2}}{2\left(1 / h^{2}+p_{0}\right)}
\end{array}\right]_{(N+1) \times(N+1)}, \\
& p_{0}=b_{0}-a_{0}=\frac{\tau^{-\alpha}}{(1-\alpha) \Gamma(1-\alpha)}-\frac{\tau^{-\alpha}}{(2-\alpha) \Gamma(1-\alpha)}=\frac{\tau^{-\alpha}}{\Gamma(3-\alpha)}>0 \text {, }
\end{aligned}
$$

therefore, $\rho\left(\alpha_{2}\right)<1$.

Now, assume $\rho\left(\alpha_{i}\right)<1$. After some calculations, we find that

$$
\begin{aligned}
\alpha_{i+1} & =-\left(B+A \alpha_{i}\right)^{-1} A \\
& =\left(\frac{1}{2 h^{2}}\right)\left[\begin{array}{lcccc}
0 & & & & \\
* & \frac{1}{B_{2,2}-\left(1 / 2 h^{2}\right) \alpha_{i_{2,2}}} & & & \\
* & * & \frac{1}{B_{3,3}-\left(1 / 2 h^{2}\right) \alpha_{i_{3,3}}} & & \\
* & * & * & \ddots & 1 \\
* & * & * & & \frac{1}{B_{N+1, N+1}-\left(1 / 2 h^{2}\right) \alpha_{i_{N+1, N+1}}}
\end{array}\right]
\end{aligned}
$$


and we already know that $B_{j, j}=1 / h^{2}+w_{0}$ and $\alpha_{i_{j, j}}=\rho\left(\alpha_{i}\right)$ for $2 \leq j \leq N+1$ :

$$
\rho\left(\alpha_{i+1}\right)=\left|\frac{1 / 2 h^{2}}{1 / h^{2}+p_{0}-\left(1 / 2 h^{2}\right) \rho\left(\alpha_{i}\right)}\right|=\frac{M^{2}}{2\left[M^{2}\left(1-\rho\left(\alpha_{i}\right) / 2\right)+p_{0}\right]} .
$$

Since $0 \leq \rho\left(\alpha_{i}\right)<1$, it follows that $\rho\left(\alpha_{i+1}\right)<1$. So, $\rho\left(\alpha_{i}\right)<1$ for any $i$, where $1 \leq i \leq M$.

Remark 4.1. The convergence of the method follows from the Lax equivalence theorem [31] because of the stability and consistency of the proposed scheme.

\section{Numerical Analysis}

Example 5.1. Consider

$$
\begin{gathered}
\frac{\partial^{\alpha} u(t, x)}{\partial t^{\alpha}}=\frac{\partial^{2} u(t, x)}{\partial x^{2}}+\frac{2 t^{(2-\alpha)}}{\Gamma(3-\alpha)} \sin \left(x-x^{2}\right) \\
+t^{2}\left[\sin \left(x-x^{2}\right)(1-2 x)^{2}+2 \cos \left(x-x^{2}\right)\right], \quad(0<x<1,0<t<1), \\
u(0, x)=0, \quad 0 \leq x \leq 1, \\
u(t, 0)=0, \quad u(t, 1)=0, \quad 0 \leq t \leq 1 .
\end{gathered}
$$

Exact solution of this problem is $U(t, x)=t^{2} \sin (1-x) x$. The solution by the CrankNicholson scheme is given in Figure 1. The errors when solving this problem are listed in the Table 1 for various values of time and space nodes.

The errors in the table are calculated by the formula $\max _{0 \leq n \leq M, 0 \leq k \leq N}\left|u\left(t_{k}, x_{n}\right)-U_{n}^{k}\right|$ and the error rate formula is $\left|E_{k}\right| /\left|E_{k+1}\right|$.

Example 5.2. Consider

$$
\begin{gathered}
\frac{\partial^{\alpha} u(t, x)}{\partial t^{\alpha}}=\frac{\partial^{2} u(t, x)}{\partial x^{2}}+\frac{24 t^{(2-\alpha)}}{\Gamma(5-\alpha)}\left(x^{2}-x\right)-2 t^{4}, \quad(0<x<1,0<t<1), \\
u(0, x)=0, \quad 0 \leq x \leq 1, \\
u(t, 0)=0, \quad u(t, 1)=0, \quad 0 \leq t \leq 1 .
\end{gathered}
$$

Exact solution of this problem is $U(t, x)=t^{4} x(x-1)$. The solution by the CrankNicholson scheme is given in Figure 2. The errors when solving this problem are listed in Table 2 for various values of time and space nodes and several values of $\alpha$.

It can be concluded from the tables and the figures that when the step size is reduced by a factor of $1 / 2$, the error decreases by about $1 / 4$. The numerical results support the claim about the order of the convergence. 


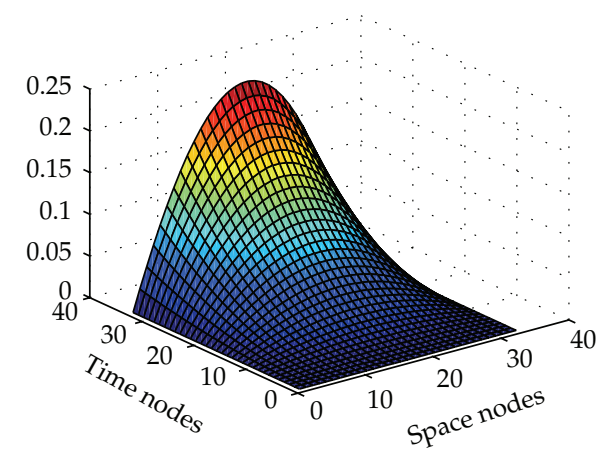

(a)

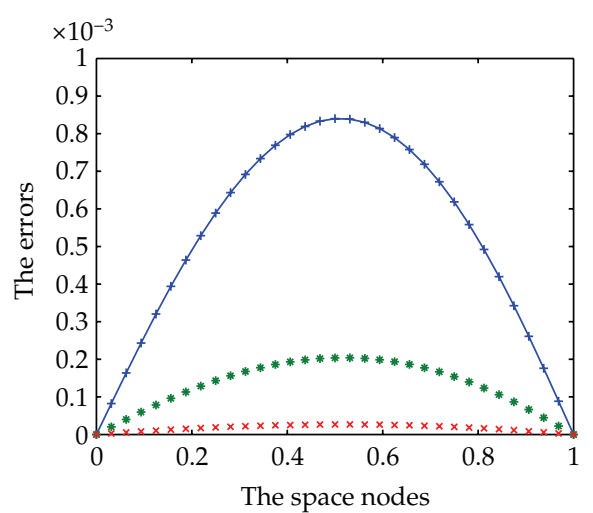

$$
\begin{aligned}
+\quad N=8, M=32 \\
* \quad N=16, M=32 \\
\times \quad N=32, M=32
\end{aligned}
$$

(b)

Figure 1: (a) The approximate solutions of Example 5.1 by the proposed method when $N=32, M=32$, and $\alpha=0.5$. (b) The errors for some values of $M$ and $N$ when $t=1$ and $\alpha=0.5$.

Table 1: Error table for Example 5.1.

\begin{tabular}{cccccccc}
\hline & \multicolumn{2}{c}{$\alpha=0.2$} & \multicolumn{2}{c}{$\alpha=0.5$} & \multicolumn{2}{c}{$\alpha=0.9$} \\
$M$ & $N$ & Error & Rate & Error & Rate & Error & Rate \\
\hline 32 & 8 & 0.0018870311 & - & 0.0016846217 & - & 0.0009754809 & - \\
32 & 16 & 0.0004703510 & 4.01 & 0.0004052354 & 4.16 & 0.0002461078 & 3.97 \\
32 & 32 & 0.0001172029 & 4.01 & 0.0000969929 & 4.18 & 0.0000650942 & 3.78 \\
32 & 64 & 0.0000291961 & 4.01 & 0.00002314510 & 4.19 & 0.0000198362 & 3.28 \\
\hline
\end{tabular}

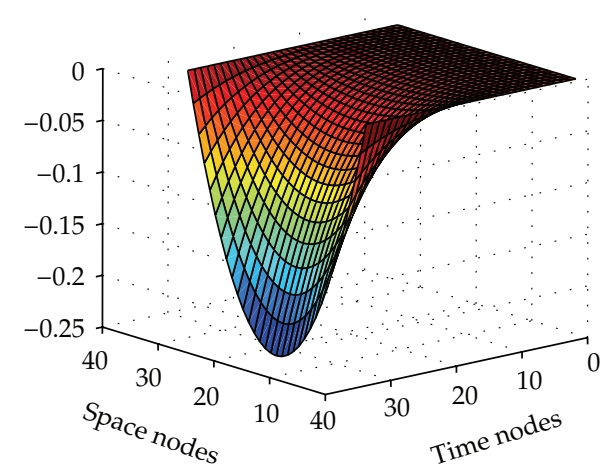

(a)

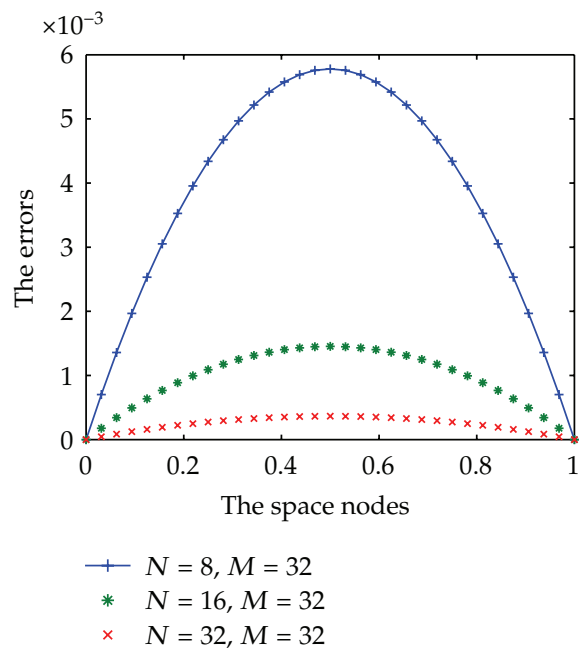

(b)

Figure 2: (a) The approximate solutions of Example 5.2 by the proposed method when $N=32, M=32$, and $\alpha=0.5$. (b) The errors for some values of $M$ and $N$ when $t=1$ and $\alpha=0.5$. 
Table 2: The errors for some values of $M, N$, and $\alpha$.

\begin{tabular}{lccccccc}
\hline & \multicolumn{2}{c}{$\alpha=0.3$} & \multicolumn{2}{c}{$\alpha=0.5$} & \multicolumn{2}{c}{$\alpha=0.8$} \\
$M$ & $N$ & Error & Rate & Error & Rate & Error & Rate \\
\hline 4 & 4 & 0.02321328680 & - & 0.02286737567 & - & 0.02173420667 & - \\
8 & 8 & 0.00583004420 & 3.98 & 0.00577931685 & 3.96 & 0.00554721754 & 3.92 \\
16 & 16 & 0.00146112785 & 3.99 & 0.00145293106 & 3.98 & 0.00140076083 & 3.96 \\
32 & 32 & 0.00036572715 & 3.995 & 0.00036424786 & 3.99 & 0.00035252421 & 3.97 \\
64 & 64 & 0.00009148685 & 3.998 & 0.00009122231 & 3.99 & 0.00008860379 & 3.98 \\
\hline
\end{tabular}

\section{Conclusion}

In this work, the Crank-Nicholson difference scheme was successfully extended to solve the time-fractional heat equations. A second-order approximation for the Riemann-Liouville fractional derivative is obtained. It is proven that the time-fractional Crank-Nicholson difference scheme is unconditionally stable and convergent. Numerical results are in good agreement with the theoretical results.

\section{References}

[1] X. Li, X. Han, and X. Wang, "Numerical modeling of viscoelastic flows using equal low-order finite elements," Computer Methods in Applied Mechanics and Engineering, vol. 199, no. 9-12, pp. 570-581, 2010.

[2] R. L. Bagley and P. J. Torvik, "Theoretical basis for the application of fractional calculus to viscoelasticity," Journal of Rheology, vol. 27, no. 3, pp. 201-210, 1983.

[3] M. Raberto, E. Scalas, and F. Mainardi, "Waiting-times and returns in high-frequency financial data: an empirical study," Physica A, vol. 314, no. 1-4, pp. 749-755, 2002.

[4] E. Scalas, R. Gorenflo, and F. Mainardi, "Fractional calculus and continuous-time finance," Physica A, vol. 284, no. 1-4, pp. 376-384, 2000.

[5] D. A. Benson, S. W. Wheatcraft, and M. M. Meerschaert, "Application of a fractional advection-dispersion equation," Water Resources Research, vol. 36, no. 6, pp. 1403-1412, 2000.

[6] L. Galue, S. L. Kalla, and B. N. Al-Saqabi, "Fractional extensions of the temperature field problems in oil strata," Applied Mathematics and Computation, vol. 186, no. 1, pp. 35-44, 2007.

[7] I. Podlubny, Fractional Differential Equations: An introduction to Fractional Derivatives, Fractional Differential Equations, to Methods of Their Solution and Some of Their Applications, vol. 198 of Mathematics in Science and Engineering, Academic Press, San Diego, Calif, USA, 1999.

[8] X. Li, M. Xu, and X. Jiang, "Homotopy perturbation method to time-fractional diffusion equation with a moving boundary condition," Applied Mathematics and Computation, vol. 208, no. 2, pp. 434 $439,2009$.

[9] J. A. T. Machado, “Discrete-time fractional-order controllers," Fractional Calculus \& Applied Analysis, vol. 4, no. 1, pp. 47-66, 2001.

[10] O. P. Agrawal, O. Defterli, and D. Baleanu, "Fractional optimal control problems with several state and control variables," Journal of Vibration and Control, vol. 16, no. 13, pp. 1967-1976, 2010.

[11] D. Baleanu, O. Defterli, and O. P. Agrawal, "A central difference numerical scheme for fractional optimal control problems," Journal of Vibration and Control, vol. 15, no. 4, pp. 583-597, 2009.

[12] Z. M. Odibat and S. Momani, "Application of variational iteration method to nonlinear differential equations of fractional order," International Journal of Nonlinear Sciences and Numerical Simulation, vol. 7, no. 1, pp. 27-34, 2006.

[13] Z. Odibat and S. Momani, "Numerical methods for nonlinear partial differential equations of fractional order," Applied Mathematical Modelling, vol. 32, no. 1, pp. 28-39, 2008.

[14] S. Momani and Z. Odibat, "Analytical solution of a time-fractional Navier-Stokes equation by Adomian decomposition method," Applied Mathematics and Computation, vol. 177, no. 2, pp. 488-494, 2006. 
[15] Z. M. Odibat and S. Momani, "Approximate solutions for boundary value problems of time-fractional wave equation," Applied Mathematics and Computation, vol. 181, no. 1, pp. 767-774, 2006.

[16] S. S. Ray and R. K. Bera, "Analytical solution of a fractional diffusion equation by Adomian decomposition method," Applied Mathematics and Computation, vol. 174, no. 1, pp. 329-336, 2006.

[17] I. Podlubny, The Laplace Transform Method for Linear Differential Equations of Fractional Order, Slovac Academy of Science, Bratislava, Slovakia, 1994.

[18] B. Baeumer, D. A. Benson, and M. M. Meerschaert, "Advection and dispersion in time and space," Physica A, vol. 350, no. 2-4, pp. 245-262, 2005.

[19] Z. Q. Deng, V. P. Singh, and L. Bengtsson, "Numerical solution of fractional advection-dispersion equation," Journal of Hydraulic Engineering, vol. 130, no. 5, pp. 422-431, 2004.

[20] V. E. Lynch, B. A. Carreras, D. del-Castillo-Negrete, K. M. Ferreira-Mejias, and H. R. Hicks, "Numerical methods for the solution of partial differential equations of fractional order," Journal of Computational Physics, vol. 192, no. 2, pp. 406-421, 2003.

[21] M. M. Meerschaert and C. Tadjeran, "Finite difference approximations for two-sided space-fractional partial differential equations," Applied Numerical Mathematics, vol. 56, no. 1, pp. 80-90, 2006.

[22] M. M. Meerschaert and C. Tadjeran, "Finite difference approximations for fractional advectiondispersion flow equations," Journal of Computational and Applied Mathematics, vol. 172, no. 1, pp. 65-77, 2004.

[23] I. Karatay, S. R. Bayramoğlu, and A. Şahin, "Implicit difference approximation for the time fractional heat equation with the nonlocal condition," Applied Numerical Mathematics, vol. 61, no. 12, pp. 12811288, 2011.

[24] D. Baleanu, K. Diethelm, E. Scalas, and J. J. Trujillo, Fractional Calculus: Models and Numerical Methods, vol. 3 of Series on Complexity, Nonlinearity and Chaos, World Scientific Publishing, Hackensack, NJ, USA, 2012.

[25] M. M. Meerschaert, H.-P. Scheffler, and C. Tadjeran, "Finite difference methods for two-dimensional fractional dispersion equation," Journal of Computational Physics, vol. 211, no. 1, pp. 249-261, 2006.

[26] C. Tadjeran, M. M. Meerschaert, and H.-P. Scheffler, "A second-order accurate numerical approximation for the fractional diffusion equation," Journal of Computational Physics, vol. 213, no. 1, pp. 205-213, 2006.

[27] L. Su, W. Wang, and Z. Yang, "Finite difference approximations for the fractional advection-diffusion equation," Physics Letters A, vol. 373, no. 48, pp. 4405-4408, 2009.

[28] A. M. Abu-Saman and A. M. Assaf, "Stability and convergence of Crank-Nicholson method for fractional advection dispersion equation," Advances in Applied Mathematical Analysis, vol. 2, no. 2, pp. 117-125, 2007.

[29] G. Jumarie, "Modified Riemann-Liouville derivative and fractional Taylor series of nondifferentiable functions further results," Computers \& Mathematics with Applications, vol. 51, no. 9-10, pp. 1367-1376, 2006.

[30] S. Zhang, "Monotone iterative method for initial value problem involving Riemann-Liouville fractional derivatives," Nonlinear Analysis: Theory, Methods E Applications, vol. 71, no. 5-6, pp. 2087-2093, 2009.

[31] R. D. Richtmyer and K. W. Morton, Difference Methods for Initial-Value Problems, Interscience Publishers, New York, NY, USA, 1967. 


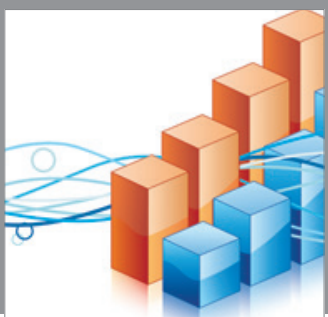

Advances in

Operations Research

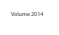

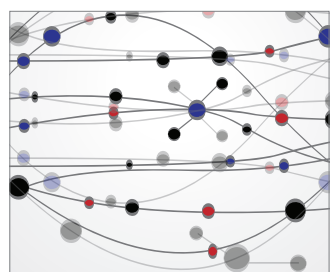

\section{The Scientific} World Journal
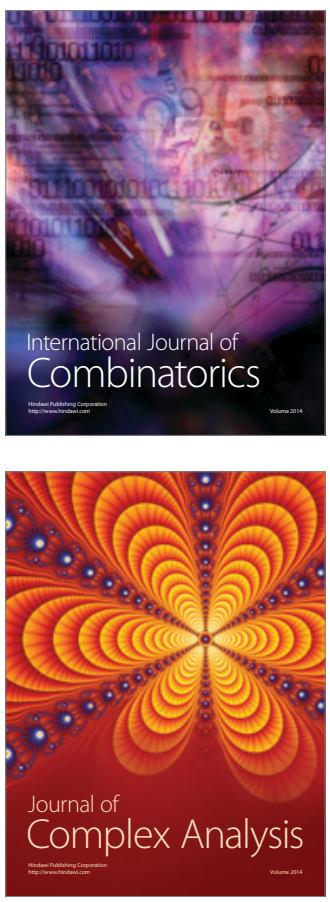

International Journal of

Mathematics and

Mathematical

Sciences
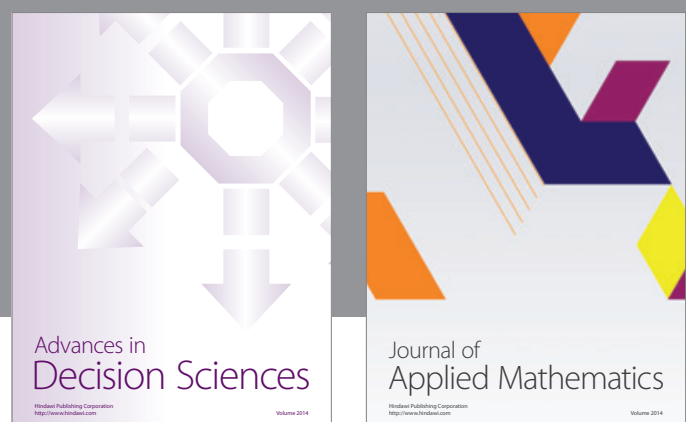

Journal of

Applied Mathematics
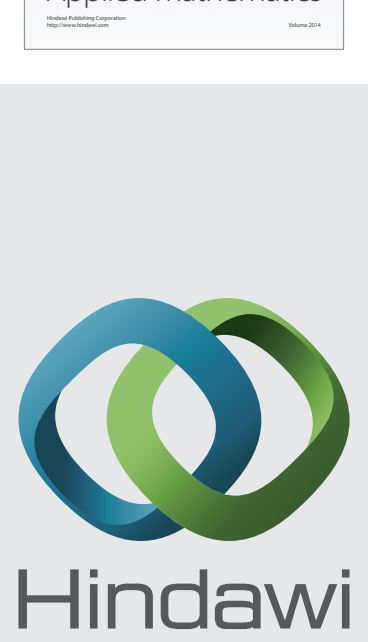

Submit your manuscripts at http://www.hindawi.com
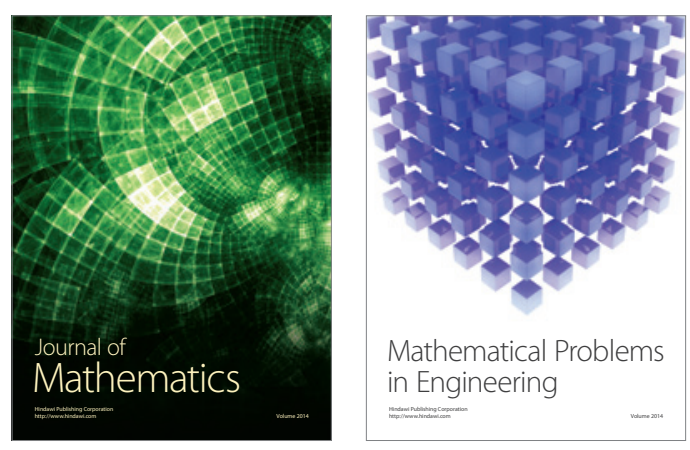

Mathematical Problems in Engineering
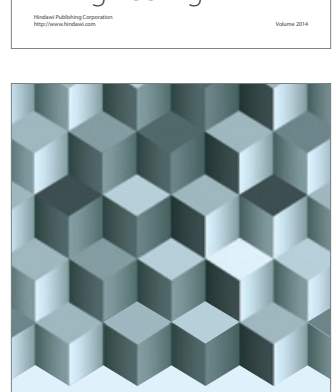

Journal of

Function Spaces
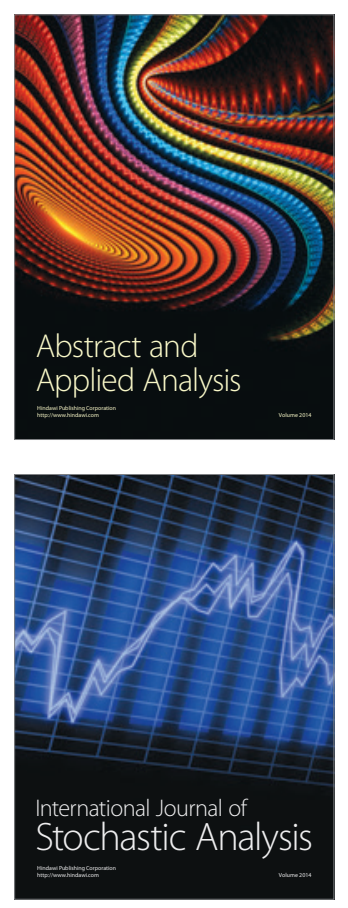

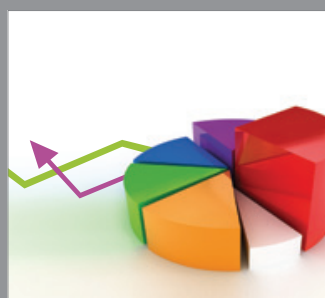

ournal of

Probability and Statistics

Promensencen
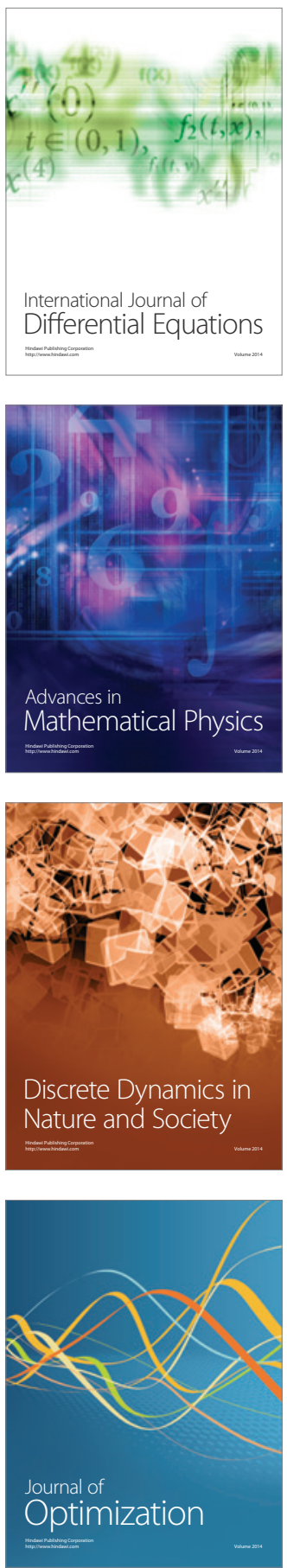